\title{
ISOMETRIC EMBEDDINGS OF KÄHLER-RICCI SOLITONS IN THE COMPLEX PROJECTIVE SPACE
}

\author{
LUCIO BEDULLI AND ANNA GORI
}

(Communicated by Lei $\mathrm{Ni}$ )

\begin{abstract}
We prove that a compact complex manifold endowed with a nontrivial Kähler-Ricci soliton cannot be isometrically embedded in the FubiniStudy complex projective space as a complete intersection.
\end{abstract}

\section{INTRODUCTION}

A Kähler metric $g$ on a complex manifold $M$ is said to be a Kähler-Ricci soliton if there exists a holomorphic vector field $V$ on $M$ such that

$$
\operatorname{Ric}(g)=\lambda g+\mathcal{L}_{V} g,
$$

where $\lambda$ is a real constant. Kähler-Ricci solitons have been extensively studied in recent years mainly because they provide self-similar solutions to the Kähler-Ricci flow which was introduced as a means for finding Kähler-Einstein metrics. KählerRicci solitons are indeed a generalization of Kähler-Einstein metrics (taking $V=0$ in (0.1) we get the Einstein equation), but they are an alternative to them because the presence of a Kähler-Ricci soliton with nontrivial $V$ is an obstruction to the existence of a Kähler-Einstein metric on a compact complex manifold with positive first Chern class (the Futaki invariant with respect to the real part of $V$ is nonzero). In fact it is a deep result proved by Tian and Zhu [10] that a compact Fano manifold can admit at most one Kähler-Ricci soliton, including trivial ones.

The first nontrivial examples of compact Kähler-Ricci solitons were found by Koiso: in [5] he proved the existence of a KRS on any Fano manifold admitting a cohomogeneity one action of a compact semisimple Lie group of isometries with two complex singular orbits. After that, Wang and Zhu 11] proved the existence of a KRS on any compact toric Fano manifold, and this result was later generalized in [8] to toric bundles over generalized flag manifolds. Since all compact KRS are Fano and can be holomorphically embedded in the complex projective space $\mathbb{C P}^{m}$, it is natural to ask whether a Kähler-Ricci soliton may be induced by the Fubini-Study metric of $\mathbb{C P}^{m}$.

In this note we prove the following negative result. Recall that a smooth codimension $r$ subvariety of $\mathbb{C P}^{m}$ is a complete intersection if its ideal is generated by $r$ elements or equivalently if it may be described as the transverse intersection of $r$ algebraic hypersurfaces.

Received by the editors June 27, 2012.

2010 Mathematics Subject Classification. Primary 32Q20, 53C25, 53C55. 
Theorem 0.1. Let $M$ be a closed complex submanifold of $\mathbb{C P}^{m}$ such that the metric induced on $M$ by the Fubini-Study metric $\omega_{F S}$ is a Kähler-Ricci soliton. If $M$ is a complete intersection, then the Kähler-Ricci soliton is trivial and $M$ is a linear subspace or a smooth quadric subvariety of some linear subspace.

Our result may be thought of as a generalization of the main theorem of [3], where the classification of Kähler-Einstein manifolds isometrically embedded in $\left(\mathbb{C P}^{n}, \omega_{F S}\right)$ as complete intersections is given. For general smooth subvarieties, beside the homogeneous case of flag manifolds (see [9] for the classification), no example of a positive Kähler-Einstein metric induced by $\omega_{F S}$ is known. On the other hand, a Kähler-Einstein submanifold of $\left(\mathbb{C P}^{n}, \omega_{F S}\right)$ has necessarily positive scalar curvature by a result of Hulin [4].

\section{Proof of the theorem}

1.1. Kähler-Ricci solitons. Let $M$ be a complex manifold and denote by $J$ its complex structure. Rephrasing (0.1) in terms of 2-forms, a Kähler-Ricci soliton on $M$ is a Kähler metric $g$ whose associated Ricci and Kähler forms $\rho$ and $\omega=g(J \cdot, \cdot)$ respectively satisfy

$$
\rho=\lambda \omega+\mathcal{L}_{V} \omega
$$

for some holomorphic vector field $V=X-i J X$, where $J$ is the complex structure. We will say that the Kähler-Ricci soliton is trivial if $V=0$; i.e. $(M, g)$ is KählerEinstein.

Note that $\mathcal{L}_{X} J=0$ because $V$ is holomorphic and equation (1.1) implies that $\mathcal{L}_{J X} \omega=0$; i.e. $J X$ preserves $\omega$, hence $g$, because it also preserves $J$. Note also that (1.1) implies

$$
\rho=\lambda \omega+\mathcal{L}_{X} \omega
$$

The fact that $\nabla X$ is $g$-self-adjoint means that the 1-form dual to $X$ is closed. Since a KRS may exist only on Fano manifolds and these are simply connected (Kobayashi's theorem), we see that $X$ is the gradient with respect to $g$ of some smooth function $f$. We will indicate $\nabla f:=\operatorname{grad}_{g}(f)$. This implies that

$$
\mathcal{L}_{X} \omega=\mathcal{L}_{\nabla f} \omega=d \iota_{\nabla f} \omega=d d^{c} f .
$$

Recalling 1 that $\partial=\frac{1}{2}\left(d+i d^{c}\right)$ and $\bar{\partial}=\frac{1}{2}\left(d-i d^{c}\right)$, equation (1.2) turns out to be equivalent to

$$
\rho=\lambda \omega+2 i \partial \bar{\partial} f .
$$

Indeed the previous computation also shows that the function $f$ indeed admits another useful interpretation. Since $\iota_{J X} \omega=-d f$ the function $f$ is, up to a constant multiple, a moment map for the infinitesimal action of the Killing vector field $J X$ on $M$, or, more precisely, it is the projection along $J X$ of a moment map $\mu$ for the Hamiltonian action of Iso(M,g) on $M$. (Recall that since $M$ is simply connected, every symplectic action on $M$ is Hamiltonian.)

\footnotetext{
${ }^{1}$ We are using the convention according to which $d^{c} h(Y)=J d h(Y)=d h(-J Y)$.
} 
1.2. Proof of the theorem. Let $n$ be the complex dimension of $M$ and $r=m-n$ the codimension. Denote also by $i: M \rightarrow \mathbb{C P}^{m}$ the inclusion and simply by $\omega$ the restriction $i^{*} \omega_{F S}$. By hypothesis $\omega$ satisfies (1.3) where $f$ is the potential of the holomorphic vector field $X=\nabla f$. We suppose that $M$ is embedded in $\mathbb{C P}^{m}$ as a complete intersection. Namely $M$ is assumed to admit $r$ homogeneous polynomials $P_{1}, P_{2}, \ldots, P_{r}$ on $\mathbb{C}^{m+1}$ which define $M$ as their zero locus and generate the ideal associated to $M$.

It is a direct consequence of the adjunction formula that the canonical line bundle $K_{M}=\Lambda^{n, 0} M$ of $M$ is the restriction of a line bundle on $\mathbb{C P}^{m} ;$ more precisely,

$$
K_{M}=i^{*} \mathcal{O}(d-m-1),
$$

where $d=\sum_{j=1}^{r} \operatorname{deg} P_{j}$. Since the Chern class of $K_{M}$ is represented by $\frac{1}{2 \pi}$ times the Ricci form, the constant $\lambda$ in (1.3) is forced to be equal to $m+1-d>0$.

It is well known that Hermitian metrics $h$ on $K_{M}^{*}$ correspond bijectively to positive volumes (nowhere vanishing real $2 n$-forms) $v$ of $M$, the correspondence being given by

$$
\left\langle v,(-2)^{m}(\sqrt{-1})^{m^{2}} x \wedge \bar{x}\right\rangle=h(x, x)
$$

for $x \in K_{M}^{*}$. Let $V$ be the volume of $M$ corresponding to the fibre metric on $K_{M}^{*}$ whose Chern curvature form is exactly $(m+1-d) \omega$. In [3] (Proposition 2) the real positive function $\phi$ is computed explicitly such that $\omega^{n}=\phi V$ in the case where $M$ is a complete intersection. More precisely, recalling that the Chern curvature form of the fibre metric induced by $\omega$ on $K_{M}^{*}$ is exactly the Ricci form $\rho$ (see [1, p. 82), we have the following.

Proposition 1.1 (Hano [3]). Let $M$ be a complete intersection in $\mathbb{C P}^{m}$ defined by the polynomials $P_{1}, \ldots, P_{r}$. Denote by $d=\sum_{i} \operatorname{deg} P_{i}$ and by $\rho$ the Ricci form of the metric $\omega$ induced by $\omega_{F S}$. Then we have

$$
\rho=(m+1-d) \omega+i \partial \bar{\partial} \log \phi, \quad \text { with } \quad \phi=\frac{\left\|d P_{1} \wedge d P_{2} \wedge \cdots \wedge d P_{r}\right\|^{2}}{\|z\|^{2(d-r)}} .
$$

Here $\phi$ is expressed in terms of unitary homogeneous coordinates of $\mathbb{C P}^{m}$ and $\left\|d P_{1} \wedge d P_{2} \wedge \cdots \wedge d P_{r}\right\|^{2}=\sum\left|P_{\lambda_{1} \cdots \lambda_{r}}\right|^{2}$, where $d P_{1} \wedge d P_{2} \wedge \cdots \wedge d P_{r}=\sum P_{\lambda_{1} \cdots \lambda_{r}} d z_{\lambda_{1}} \wedge$ $\cdots \wedge d z_{\lambda_{r}}$. Note also that the previous expression of $\phi$ is invariant under any unitary coordinate transformation.

Combining (1.4) with the Kähler-Ricci soliton equation we get

$$
\partial \bar{\partial} \log \phi=2 \partial \bar{\partial} f
$$

so that

$$
\phi=C \cdot e^{2 f}
$$

for some constant $C \in \mathbb{R}$. Now the key fact is that we can also find an explicit expression for $f$ in terms of homogeneous coordinates of $\mathbb{C P}^{m}$. Indeed, as already remarked, $f$ is a moment map for the action of the 1-parameter group of isometries generated by $J X$, and this enables us to write it in suitable coordinates. To start with, by a famous result of Calabi [2] the Killing vector field $J X$ can be extended to a Killing vector field of $\left(\mathbb{C P}^{m}, \omega_{F S}\right)$ so that with respect to an appropriate system of unitary homogeneous coordinates it can be written in diagonal form, $\operatorname{diag}\left(i \lambda_{0}, \ldots, i \lambda_{m}\right)$, as an element of $\mathfrak{s u}(m+1)$. 
Thus a moment map for the Hamiltonian action of the 1-parameter group $\{\exp t J X\}$ on $\mathbb{C P}^{m}$ is

$$
\mu_{J X}=\frac{1}{2} \frac{\sum_{j=0}^{m} \lambda_{j}\left|z_{j}\right|^{2}}{\sum_{j=0}^{m}\left|z_{j}\right|^{2}},
$$

and $f$ is nothing but the restriction of $\mu_{J X}$ to $M$. So there exists a constant $C \in \mathbb{R}$ such that on $M$ one has

$$
\frac{\left\|d P_{1} \wedge d P_{2} \wedge \cdots \wedge d P_{r}\right\|^{2}}{\|z\|^{2(d-r)}}=C e^{\frac{\sum \lambda_{j}\left|z_{j}\right|^{2}}{\sum\left|z_{j}\right|^{2}}} .
$$

We claim that (1.5) holds if and only if $f(z, \bar{z})$ is constant. Let $p$ and $q$ be any two points of $M$. Since $M$ is Fano, by a theorem of Kollár Miyaoka and Mori [6] there exists a rational curve passing through $p$ and $q$, say $F: \mathbb{C P}^{1} \rightarrow M \subseteq \mathbb{C P}^{m}$, defined by $F([s: t])=\left[F_{0}(s, t): \ldots: F_{m}(s, t)\right]$ where the functions $F_{m}(s, t)$ are homogeneous polynomials of degree $\delta$ in $s$ and $t$.

Evaluating (1.5) at $F\left(\mathbb{C P}^{1}\right)$ we get

$$
\frac{\left\|d P_{1}(F([s: t])) \wedge \cdots \wedge d P_{r}(F([s: t]))\right\|^{2}}{\left(\sum_{j}\left|F_{j}(s, t)\right|^{2}\right)^{(d-r)}}=C e^{\frac{\sum \lambda_{j}\left|F_{j}(s, t)\right|^{2}}{\sum\left|F_{j}(s, t)\right|^{2}}}
$$

for every $[s: t] \in \mathbb{C P}^{1}$, and this is clearly impossible unless $f$ is constant on $F\left(\mathbb{C P}^{1}\right)$; otherwise the right hand side of (1.6) would not be a rational function of $s$ and $t$. Since $p$ and $q$ are arbitrary, $f$ must be constant on all of $M$ : this means that $X=\nabla f$ vanishes and the Kähler-Ricci soliton is trivial; i.e. $\omega$ is Kähler-Einstein. According to Hano [3] this happens only if $M$ is a linear subspace or it is a smooth quadric subvariety of some linear subspace.

\section{RefERENCES}

[1] Arthur L. Besse, Einstein manifolds, Ergebnisse der Mathematik und ihrer Grenzgebiete (3) [Results in Mathematics and Related Areas (3)], vol. 10, Springer-Verlag, Berlin, 1987. MR867684 (88f:53087)

[2] Eugenio Calabi, Isometric imbedding of complex manifolds, Ann. of Math. (2) 58 (1953), 1-23. MR0057000 (15,160c)

[3] Jun-ichi Hano, Einstein complete intersections in complex projective space, Math. Ann. 216 (1975), no. 3, 197-208. MR0382704 (52 \#3586)

[4] Dominique Hulin, Kähler-Einstein metrics and projective embeddings, J. Geom. Anal. 10 (2000), no. 3, 525-528, DOI 10.1007/BF02921947. MR.1794575 (2001k:32042)

[5] Norihito Koiso, On rotationally symmetric Hamilton's equation for Kähler-Einstein metrics, Recent topics in differential and analytic geometry, Adv. Stud. Pure Math., vol. 18, Academic Press, Boston, MA, 1990, pp. 327-337. MR1145263 (93d:53057)

[6] János Kollár, Yoichi Miyaoka, and Shigefumi Mori, Rational connectedness and boundedness of Fano manifolds, J. Differential Geom. 36 (1992), no. 3, 765-779. MR.1189503 (94g:14021)

[7] Zhiqin Lu, On the Futaki invariants of complete intersections, Duke Math. J. 100 (1999), no. 2, 359-372, DOI 10.1215/S0012-7094-99-10013-5. MR.1722959 (2000h:32033)

[8] Fabio Podestà and Andrea Spiro, Kähler-Ricci solitons on homogeneous toric bundles, J. Reine Angew. Math. 642 (2010), 109-127, DOI 10.1515/CRELLE.2010.038. MR2658183 (2011f:32049)

[9] Masaru Takeuchi, Homogeneous Kähler submanifolds in complex projective spaces, Japan. J. Math. (N.S.) 4 (1978), no. 1, 171-219. MR528871 (80i:32059) 
[10] Gang Tian and Xiaohua Zhu, Uniqueness of Kähler-Ricci solitons, Acta Math. 184 (2000), no. 2, 271-305, DOI 10.1007/BF02392630. MR1768112(2001h:32040)

[11] Xu-Jia Wang and Xiaohua Zhu, Kähler-Ricci solitons on toric manifolds with positive first Chern class, Adv. Math. 188 (2004), no. 1, 87-103, DOI 10.1016/j.aim.2003.09.009. MR:2084775 (2005d:53074)

Dipartimento di Matematica, Università dell' Aquila, via Vetoio loc. Coppito, 67100 L'AQUila, ITALY

E-mail address: lucio.bedulli@dm.univaq.it

Dipartimento di Matematica, Università di Milano, Via Saldini 50, 20133 Milano, ITALY

E-mail address: anna.gori@unimi.it 